\title{
New opinion on the subtypes of poststroke depression in Chinese stroke survivors
}

\author{
This article was published in the following Dove Press journal: \\ Neuropsychiatric Disease and Treatment \\ 6 March 2017 \\ Number of times this article has been viewed
}

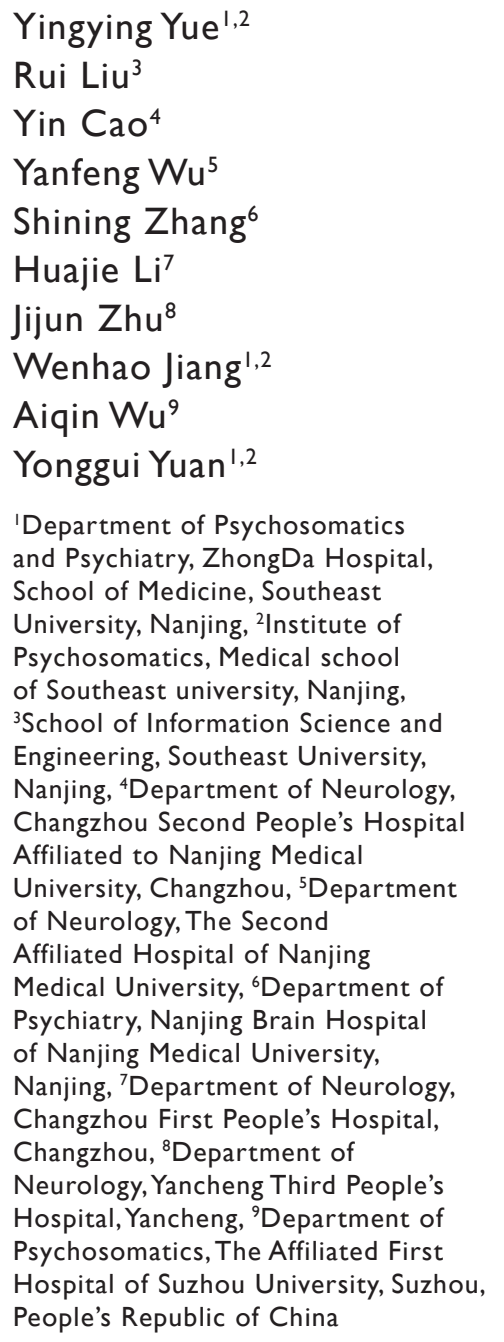

Correspondence: Yonggui Yuan Department of Psychosomatics and Psychiatry, ZhongDa Hospital, School of Medicine, Southeast University, No 87 Dingjiaqiao Road, Nanjing 210009 , People's Republic of China Tel +860258328 5I 24 Email yygylh2000@sina.com

\begin{abstract}
Aim: Poststroke depression (PSD) is the most common complication of stroke. However, some stroke survivors with depression cannot meet the diagnostic criteria of PSD. The aim of this study was to propose the new conception of stroke patients with depression and then make them to receive reasonable diagnosis and treatment.

Methods: We first put forward the opinion that the general PSD should consist of PSD disorder (PSDD) and PSD symptoms (PSDS) according to the Diagnostic and Statistical Manual of Mental Disorder - Fifth Edition (DSM-5) and ZhongDa diagnostic criteria - first edition (ZD-1), respectively. The ZD-1 was established based on the suggestions of 65 Chinese chief doctors considering that the symptoms of PSDS might be different from those of PSDD and the duration of DSM-5 was too strict. Then, 166 stroke inpatients were recruited, and the study was conducted using the diagnosis and classification of PSD to verify the new concept.
\end{abstract}

Results: A total of 24 (14.46\%) and 80 (48.19\%) stroke patients were diagnosed with PSDD and PSDS, respectively, according to individual diagnosis criteria. Moreover, patients meeting the diagnostic criteria of PSDD should satisfy the criteria of PSDS first. The distribution frequencies of depressive symptoms were different, which suggested that there might be discrepant depressive symptoms between PSDS and PSDD.

Conclusion: The present study proposes new opinion about the classification and diagnosis of depression in stroke survivors. The definition and criteria of PSDS are beneficial to explore phenomenological consistency and provide useful information for early recognition and appropriate interventions.

Keywords: poststroke depression, subtypes, diagnostic criteria

\section{Introduction}

Poststroke depression (PSD) is a common complication and is associated with increased physical disability, poor functional outcome and increased mortality. ${ }^{1,2}$ It is very important to establish early recognition and rational diagnosis for this disease. However, studies on PSD have primarily focused on exploring its prevalence, pathogenic factors as well as treatment. ${ }^{3-7}$ Only a few researchers have pursued the psychopathology and diagnosis of depression after stroke. ${ }^{8,9}$ Like other psychiatric disorders, the diagnostic nosology was compelled to focus on clinical manifestations, duration and function disrupted due to ambiguous etiology. However, there are no accurate diagnostic and classified criteria in the following three diagnosis systems: the Diagnostic and Statistical Manual of Mental Disorder - Fifth Edition (DSM-5), the International Classification of Disease, Tenth Edition, and Chinese Classification of Mental Disorders, Third Version. It was also just described as "depressive disorder due to another medical condition” in the DSM-5 published in May 2013 (293.83). 
Regarding classification, some researchers put forward two forms of PSD, which are major and minor depression. The major depression is defined as MDD, while the minor depression is less well defined and usually diagnosed by dysthymic disorder. ${ }^{10}$

Previous results reported that the prevalence of PSD is $5 \%-67 \%$ among all types of stroke patients. ${ }^{11}$ This variation is influenced by the diagnostic criteria, evaluation instruments, different time intervals after the stroke, varied study populations, as well as the environment (hospital or community). ${ }^{12,13}$ Among them, the nonstandard diagnostic criteria applied remain the main reasons for this broad range. The majority of previous studies used the contemporary diagnostic criteria of DSM-III/IV and the structured clinical interviews, ${ }^{14}$ while other studies used assessment scale as diagnostic criteria, which might be inappropriate. ${ }^{15,16}$

However, questions remain whether the same diagnostic criteria used in primary major depressive patients should be used in stroke survivors. First of all, the DSM-5 requires more than five depressive symptoms, with at least one of the symptoms being either 1) depressed mood or 2) loss of interest or pleasure. Many depressed stroke patients will be rejected by using such strict demands. In the second place, some symptoms of diagnosing depression may occur in physically ill patients without depression ${ }^{17}$ or some patients deny symptoms of depression due to anosognosia, cognitive dysfunction, neglect and pathological emotionalism. ${ }^{18}$ Third, DSM-5 claims 2-week course of the depressive symptoms to diagnose major depressive disorder (MDD). It may miss the best observation period because average days of hospitalization were seven to ten in the Department of Neurology. For these reasons, some researchers debated that there is overdiagnosis or misdiagnosis using DSM as criteria to diagnose depression in stroke patients. ${ }^{8,19}$ Therefore, we assume that the generalized PSD should include PSD symptoms (PSDS) and PSD disorder (PSDD) with different depressive characters, diagnostic criteria and treatment strategy. PSDS is the transition between normal and earliest manifestations of MDD, which describes the depressive state of individuals who do not fulfill MDD of the DSM-5 criteria. We further propose the ZhongDa diagnostic criteria - first edition (ZD-1) of PSDS based on the assessment scale (detailed in the "Methods" section). ${ }^{20}$ Then, the individuals diagnosed with PSDS initiate antidepressant therapy to avoid the depressive progression and deterioration, while PSDD is considered as MDD after stroke.

The experience accumulated in the past few decades supports the notion that PSD is by no means a synonym of primary depression. Considering the insidious and progressive nature of mood disorders, we can put forward a new classification for PSD. The aim of the present study was to discuss the conceptual and practical aspects of diagnosis, assessment strategies and short- and long-term prognosis associated with different types of PSD.

\section{Methods}

\section{Samples}

Stroke inpatients were recruited from March 2015 to October 2015 in the Jiangsu province. All subjects provided written informed consent to participate in this study. The study was approved by the Medical Ethics Committee for Clinical Research of ZhongDa Hospital affiliated to Southeast University. A total of 166 patients who fulfilled the following criteria were recruited: 1) participants with ischemic stroke and intracerebral hemorrhage determined by computed tomography (CT) or magnetic resonance imaging (MRI); 2) participants evaluated with patient health questionnaire-9 (PHQ-9), PSD scale (PSD-S), mini-mental state examination (MMSE), National Institutes of Health Stroke Scale (NIHSS), modified Rankin scale (mRS), Barthel index (BI); 3) participants who were antidepressant-naïve; 4) participants free of depressive episode before stroke and other major psychiatric disorders, including schizophrenia, bipolar disorder, substance abuse (caffeine, nicotine and alcohol), neurodegenerative illness, severe physical illnesses and other medical illnesses; 5) participants free of vision, hearing and memory disorders and other symptoms that hindered assessment.

\section{Classification and diagnosis of PSD}

We put forward that the general PSD should include PSDS and PSDD according to each diagnostic criteria. The ZD-1 was established for PSDS considering the following problems. First, the symptoms of PSDS might be different from MDD, so we revised the symptoms in the diagnostic criteria for MDD in DSM-5 based on the suggestions of 65 Chinese chief doctors, keeping five general symptoms (easy to cry, insomnia, easy fatigability, feeling of decreased capability and suicidal ideation) and adding three specific symptoms (decreased speech, feeling of difficult to recover, more irritable than usual). Second, considering the incipient depression of PSD and the short hospitalization period of acute stroke patients, the course was adjusted to 1 week for early recognition of PSDS. Finally, we establish the diagnostic criteria of PSDS (Table 1) on the basis of the diagnostic framework for psychiatric disease. The differences between DSM-5 and 
Table I Diagnostic criteria of ZhongDa (first edition) for PSDS

A. Three (or more) of the following symptoms have been present during the same I-week period and represent a change from previous functioning.

I. Decreased speech (eg, do not want to speak) most of the day, daily or most of the week.

2. Fatigue or loss of energy daily or most of the week.

3. Depressed mood persisting through the day, daily or most of the week, as indicated by either self-report or observation made by others (eg, feels sad, easy to cry).

4. Insomnia, wake up early or hypersomnia daily or most of the week.

5. Feelings of decreased capability, worthlessness most of the day, daily or most of the week.

6. Recurrent thoughts of death (not just fear of dying), recurrent suicidal ideation without a specific plan or a suicide attempt or a specific plan for committing suicide.

7. Feeling of hopelessness or despair (especially feeling hard to recover from the disease) most of the day, daily or most of the week.

8. More irritable than usual daily or most of the week.

B. The symptoms cause clinically significant distress or impairment in social interaction, occupation or other important areas of functioning.

C. The occurrence, development and duration of these symptoms are closely related to cerebrovascular disease.

D. The occurrence of the major depressive episode could not be better explained by adjustment disorder with depressed mood, schizoaffective disorder, schizophrenia, schizophreniform disorder, delusional disorder, or other specified and unspecified schizophrenia spectrum and other psychotic disorders.

E. No manic or a hypomanic episode is reported.

Abbreviation: PSDS, poststroke depression symptoms.

ZD-1 are listed in Table 2. All participants were carefully analyzed and diagnosed as having PSDS and PSDD according to ZD-1 and DSM- 5 criteria $^{21}$ by two trained participant neuropsychiatrists.

\section{Statistical analysis}

Data were described as mean and standard deviation (SD) for continuous variables, while classification variables were represented by number and percentage. The Statistical Package for the Social Sciences (SPSS) software version 20.0 (IBM Corp., Armonk, NY, USA) was used.

\section{Results}

\section{Demographic and neuropsychological results}

The majority of strokes $(155,93.98 \%)$ were classified as ischemic, with only 10 hemorrhagic strokes $(6.02 \%)$. Sociodemographic and clinical variables of total participants are listed in Table 3. A total of 102 males and 64 females were included in the study, with a mean age of $64.37(\mathrm{SD}=10.85)$

Table 2 Comparison of the DSM-5 and ZD-I criteria

\begin{tabular}{lll}
\hline Items & DSM-5 & ZD-I \\
\hline Depressive symptoms & & \\
Total depressive symptoms & 9 & 8 \\
Fulfilled depressive symptoms & 5 & 3 \\
Essential depressive & One of the symptoms is either & No \\
symptoms & I) depressed mood or \\
& 2) loss of interest or pleasure & \\
Duration & 2 weeks & I week \\
\hline
\end{tabular}

Abbreviations: DSM-5, Diagnostic and Statistical Manual of Mental Disorder - Fifth Edition; ZD-I, ZhongDa diagnostic criteria - first edition. and mean education level of $8.49(\mathrm{SD}=4.56)$. The mean scores of PSD-S and PHQ-9 were $6.79(\mathrm{SD}=5.61)$ and 6.60 $(\mathrm{SD}=6.07)$, respectively.

The participants had mild or moderate stroke; the mean score of NIHSS was 2.76 ( $\mathrm{SD}=2.73$ ). The mean scores of $\mathrm{mRS}$ and $\mathrm{BI}$ reflecting neurological functions were 1.81 $(\mathrm{SD}=1.28)$ and $82.95(\mathrm{SD}=22.11)$, respectively. Twenty-four $(14.46 \%)$ stroke patients had a family history of stroke.

Table 3 Demographic and neuropsychological data of all participants

\begin{tabular}{|c|c|c|c|}
\hline Item & $\begin{array}{l}\text { PSDD } \\
(n=24)\end{array}$ & $\begin{array}{l}\text { PSDS } \\
(n=80)\end{array}$ & $\begin{array}{l}\text { Total } \\
(n=166)\end{array}$ \\
\hline Age (years) & $64.50 \pm 11.26$ & $64.51 \pm 11.96$ & $64.37 \pm 10.85$ \\
\hline Gender (male/female) & $12 / 12$ & $44 / 36$ & $102 / 64$ \\
\hline Education level (years) & $8.58 \pm 6.93$ & $8.48 \pm 5.25$ & $8.49 \pm 4.56$ \\
\hline PSD-S & $|5.50 \pm 2.8|$ & $10.81 \pm 4.69$ & $6.79 \pm 5.6 \mathrm{I}$ \\
\hline PHQ-9 & $16.38 \pm 3.62$ & $10.73 \pm 5.72$ & $6.60 \pm 6.07$ \\
\hline $\begin{array}{l}\text { Stroke type } \\
\text { (ischemic/hemorrhagic) }\end{array}$ & $23 / 1$ & $73 / 7$ & $156 / 10$ \\
\hline \multicolumn{4}{|c|}{ Neurological functional assessment } \\
\hline NIHSS & $3.88 \pm 2.31$ & $3.74 \pm 2.87$ & $2.76 \pm 2.73$ \\
\hline $\mathrm{mRS}$ & $2.46 \pm 1.18$ & $2.43 \pm 1.25$ & $\mathrm{I} .8 \mathrm{I} \pm \mathrm{I} .28$ \\
\hline $\mathrm{BI}$ & $77.29 \pm 21.47$ & $75.00 \pm 23.4 I$ & $82.95 \pm 22.11$ \\
\hline \multicolumn{4}{|l|}{ Vascular risk factors, $\mathrm{n}(\%)$} \\
\hline Cardiovascular disease & $20(83.33)$ & $62(77.50)$ & $126(75.90)$ \\
\hline Metabolic diseases & $8(33.33)$ & $28(35.00)$ & $68(40.96)$ \\
\hline Active smokers & $9(37.50)$ & $35(43.75)$ & $84(50.60)$ \\
\hline Alcohol consumption & $7(29.17)$ & $19(23.75)$ & $47(28.3 I)$ \\
\hline Family history for stroke & $0(0)$ & $10(12.50)$ & $24(14.46)$ \\
\hline
\end{tabular}

Note: Data reported as mean $\pm S D$ and ratio.

Abbreviations: PSD-S, poststroke depression scale; PSDD, poststroke depression disorder; PSDS, poststroke depression symptoms; PHQ-9, patient health questionnaire-9; NIHSS, National Institutes of Health Stroke Scale; mRS, modified Rankin Scale; BI, Barthel index; SD, standard deviation. 
Table 4 Subtypes of PSD patients according to different diagnostic criteria

\begin{tabular}{llll}
\hline Subtypes of PSD & $\begin{array}{l}\text { PSDD (DSM-5 } \\
\text { diagnostic } \\
\text { criteria) }\end{array}$ & Total \\
\cline { 2 - 3 } & Yes & No & \\
\hline PSDS (ZD-I diagnostic criteria) & & & \\
Yes & 24 & 56 & 80 \\
No & 0 & 86 & 86 \\
Total & 24 & 142 & 166 \\
\hline
\end{tabular}

Abbreviations: PSD, poststroke depression; PSDD, poststroke depression disorder; DSM-5, Diagnostic and Statistical Manual of Mental Disorder - Fifth Edition; PSDS, poststroke depression symptoms; ZD-I, ZhongDa diagnostic criteria - first edition.

Of all participants, 126 (75.90\%) had cardiovascular disease and 68 (40.96\%) had metabolic disease. A total of $84(50.60 \%)$ stroke patients were active smokers, and 47 (28.31\%) participants had the habit of alcohol consumption.

\section{Subtypes of PSD diagnosed by different diagnostic criteria}

A total of 80 (48.19\%) patients were diagnosed with PSDS according to ZD-1 including 24 PSDD and 56 patients who did not meet the diagnostic criteria by DSM-5 (Table 4). The patients diagnosed with PSDS should satisfy the ZD-1 diagnostic criteria first. The depressive severity of PSDS was lower than PSDD by the PHQ-9 (10.81 \pm 4.69 vs $15.50 \pm 2.81)$ and PSD-S (10.73 \pm 5.72 vs $16.38 \pm 3.62)$.

The symptoms in the DSM-5 (nine items) and ZD-1 (eight items) have 17 items together containing three same symptoms (insomnia, waking up too early; fatigue or loss of energy; suicidal ideation). The frequency of the remaining 14 symptoms in three groups is reported in Table 5.
The depressive symptoms of first five-sevenths distribution frequency were located in ZD-1 (Decreased speech, Feeling of decreased capability, Feeling of difficult to recover, Fatigue or loss of energy and Insomnia or hypersomnia), while only two symptoms in DSM-5 ranked in first seven items (Depressed mood and Diminished interest or pleasure).

From Table 6, we can find that if we use five symptoms and 2 weeks of the ZD-1 as diagnostic criteria, 27 patients were incorporated in PSDD, which is similar to DSM-5. However, when we adopt three symptoms and 1 week as diagnostic criteria, 80 depression patients fulfilled these criteria. The detection rate of depressive patients increased from $16.27 \%$ to $48.19 \%$.

\section{Discussion}

The present study had two major findings. 1) We proposed the clear classification of PSD including PSDS and PSDD (Figure 1). PSDS described the depressive state of individuals who report emotional problems but do not reach the diagnostic criteria for MDD, which should preferably be defined and corroborated. PSDD was considered as the patients who fulfilled the criterion of major depression after stroke. 2) We highlighted the importance of formal diagnostic criteria for the purpose of identifying stroke patients who have signs of depression. The clear category provided the basis for subsequent diagnosis and treatment.

Although PSDD was widely accepted and defined in MDD criteria of DSM-IV, ${ }^{22}$ this viewpoint was argued by some investigators. ${ }^{23,24}$ In order to avoid neglecting the depressive stroke survivors down to the diagnostic criteria of MDD, Robinson et $\mathrm{a}^{25}$ suggested dividing depression into

Table 5 Symptom profile of PSD and total patients

\begin{tabular}{|c|c|c|c|}
\hline Item & $\begin{array}{l}\text { PSDD } \\
(n=24)\end{array}$ & $\begin{array}{l}\text { PSDS } \\
(\mathrm{n}=80)\end{array}$ & $\begin{array}{l}\text { Total } \\
(n=166)\end{array}$ \\
\hline Decreased speech (item I), n (\%) & $21(87.50)$ & $53(66.25)$ & $73(43.98)$ \\
\hline Easy to cry (item 2), n (\%) & $17(70.83)$ & $36(45.00)$ & $43(25.90)$ \\
\hline Feeling of decreased capability (item 3), n (\%) & $20(83.33)$ & $67(83.75)$ & $101(60.84)$ \\
\hline Feeling of difficult to recover (item 4), n (\%) & $22(91.67)$ & $56(70.00)$ & $65(39.16)$ \\
\hline More irritable than usual (item 5), n (\%) & $16(66.67)$ & $37(46.25)$ & $46(27.7 I)$ \\
\hline Fatigue or loss of energy (item 6), n (\%) & $22(91.67)$ & $68(85.00)$ & $106(63.86)$ \\
\hline Insomnia or hypersomnia (item 7), n (\%) & $22(91.67)$ & $46(57.50)$ & $61(36.75)$ \\
\hline Recurrent thoughts of death (item 8), $n$ (\%) & $8(33.33)$ & $12(15.00)$ & $14(8.43)$ \\
\hline Depressed mood (item 9), n (\%) & $24(100.00)$ & $58(72.50)$ & $76(45.78)$ \\
\hline Diminished interest or pleasure (item I0), $\mathrm{n}(\%)$ & $23(95.83)$ & $45(56.25)$ & $61(36.75)$ \\
\hline Significant weight loss when not dieting or weight gain (item II), n (\%) & $17(70.83)$ & $36(45.00)$ & $40(24.10)$ \\
\hline Psychomotor agitation or retardation (item I2), n (\%) & $13(54.17)$ & $38(47.50)$ & $50(30.12)$ \\
\hline Feelings of worthlessness or excessive or inappropriate guilt (item 13), n (\%) & $16(66.67)$ & $39(48.75)$ & $47(28.31)$ \\
\hline Diminished ability to think or concentrate, or indecisiveness (item I4), n (\%) & $18(75.00)$ & $35(43.75)$ & $52(31.33)$ \\
\hline
\end{tabular}

Abbreviations: PSD, poststroke depression; PSDD, poststroke depression disorder; PSDS, poststroke depression symptoms. 
Table 6 Patients fulfilled different depressive symptoms and duration of the ZD-I diagnostic criteria

\begin{tabular}{|c|c|c|c|}
\hline \multirow[t]{2}{*}{ Duration } & \multicolumn{3}{|c|}{ ZD-I diagnostic criteria } \\
\hline & $\begin{array}{l}\text { More than } \\
\text { five } \\
\text { symptoms }\end{array}$ & $\begin{array}{l}\text { More than } \\
\text { four } \\
\text { symptoms }\end{array}$ & $\begin{array}{l}\text { More than } \\
\text { three } \\
\text { symptoms }\end{array}$ \\
\hline More than 14 days & 27 & 31 & 36 \\
\hline $7-13$ days & 14 & 28 & 44 \\
\hline 0-6 days & 3 & 10 & 19 \\
\hline Total & 44 & 69 & 99 \\
\hline
\end{tabular}

Abbreviation: ZD-I, ZhongDa diagnostic criteria - first edition.

major and minor types according to different criteria in the 1980s. However, it will result in problems with the diagnostic criteria of dysthymia for mild PSD, such that it not only lost sight of the essential issue with distinct symptoms of these two depression types but also missed the best opportunity of clinical intervention for a standard long course. Therefore, we suggested that PSD should consist of PSDS and PSDD with different depressive characters, diagnostic criteria and treatment strategy. PSDS is the incipient depression or the earlier stages of PSD, which can progress to PSDD, maintain its original condition or alleviate by itself. At this point, it is particularly important for timely effective intervention for preventing the situation from worsening.

There are several important issues that need to be addressed in evaluating diagnostic criteria. 1) must be ascertained if these proposed modified diagnostic criteria are valid before adopting this new standard. A total

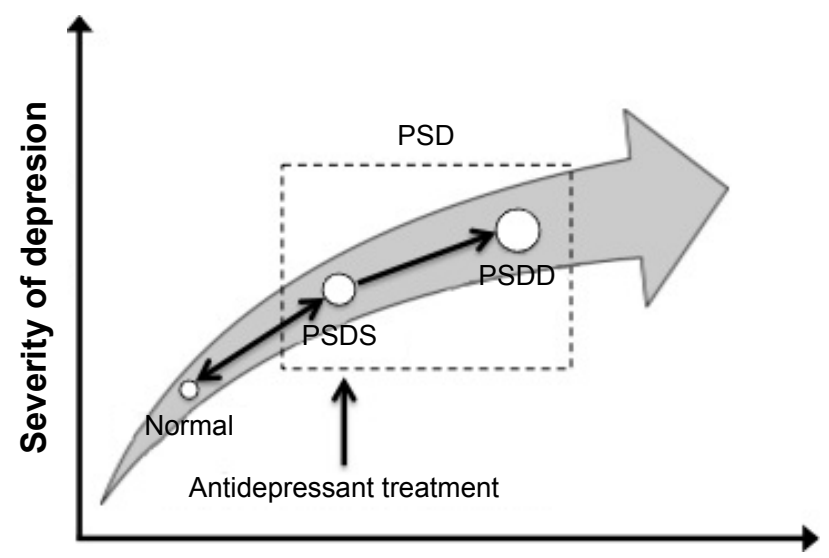

The progression of the disease

Figure I Schematic diagram for the progression of PSD

Notes: The generalized PSD should include PSDS and PSDD with different depressive characters and diagnostic criteria. PSDS is the transition between normal and PSDD, which represents the incipient depression or the earlier stages of PSD. It can progress to PSDD, maintain its original condition or alleviate by itself. The individuals diagnosed with PSDS initiate antidepressant therapy to avoid the depressive progression and deterioration, whereas PSDD is considered as MDD after stroke.

Abbreviations: PSD, poststroke depression; PSDS, poststroke depression symptoms; PSDD, poststroke depression disorder; MDD, major depressive disorder. of 27 patients were diagnosed with PSDD if we use five depressive symptoms and 2 weeks of ZD- 1 as criteria, which is similar to the existing DSM-IV criteria. This indirectly evidenced that the standards of ZD-1 were equivalent to DSM-5.

2) These criteria are based on the specific symptom or a group of phenomenological characteristics that differentiate between patients with and without the disease. Our results of five-sevenths top ranking symptoms distributing in ZD-1 manifested that the clusters of symptoms to predict PSDS are rational. Previous literature has demonstrated that crying and overt sadness are more reliable indicators of depression than apathy in the stroke unit. ${ }^{26}$ Our investigation also proved this point by prior knowledge of chief clinicians when we established the assessment scale. In spite of the fact that the two symptoms, loss of energy and feeling of difficult to recover, were also the most common characteristics in depressed and total patients, the percentages in depression group were larger than those of total participants. Similar changes were found in another study that PSD individuals have a high frequency of vegetative symptoms such as loss of energy and early awakening. ${ }^{27,28}$ Combined with this finding and previous knowledge, the ZD-1 diagnostic criteria demarcating from primary depression was good for PSDS patients. Notwithstanding, it will be better if we modulate the item of easy to cry to depressed mood covering extensive scope.

3) The course of PSDS was defined as 1 week for early recognition to facilitate the hospitalization period of acute stroke patients considering the following two reasons. On the one hand, the validity of diagnostic categories is ultimately on account of sharing similarities in etiology of all patients. The etiology of PSD is relatively specific with stroke, so we could rather easily establish the truly rational diagnosis. On the other hand, the previous study demonstrated that the prevalence of depression appeared stable during the immediate week and 3 months following the stroke, although depressive symptom profiles are likely to be different. ${ }^{29}$ We also displayed that 19 depressed stroke patients with three symptoms did not last 7 days. Further studies of the ambulatory of depression and the duration of PSDS $<7$ days are needed.

4) The diagnostic categories are the prerequisite of the treatment response and remission. Standard antidepressant treatment should be initiated once a diagnosis of PSDD was confirmed.${ }^{30}$ But today there is not enough evidence to recommend a preventive therapy for depression in any stroke patient though it shows improvement trends. ${ }^{31,32}$ Presentation of PSDS provides clear treatment indications for those patients 
with depressive symptoms. Early recognition and standard diagnosis of PSD are the preconditions of timely treatment and largest neurological and social functional recovery. ${ }^{33,34}$

Our exploratory research has several limitations. 1) Our sample was relatively small, the large population and classificatory research would be conducted in the further study. 2) The present study was a cross-sectional study; dynamic change in depression may be better presented by a follow-up study. 3) Patients included in our study were those with mild or moderate stroke, which might make a circumscribed applicability.

\section{Conclusion}

The present study put the new opinion that PSD could be divided into two types, PSDS and PSDD. PSDS is the transition between normal and PSDD, which is diagnosed by the new established ZD-1 criteria, whereas the diagnosis of PSDD is most appropriately based on DSM-5 criteria for depression due to stroke with the major depressive-like episode. Awareness of depression in stroke survivors with different subtypes represents a deep comprehension of this disease, and it is essential for early recognition, long-term management and implementation of effective interventions.

\section{Acknowledgments}

This work was supported by Jiangsu Provincial Special Program of Medical Science (BL2012025, Yonggui Yuan), The foundation for excellent doctorial dissertations of Southeast University (YBJJ1539), the Fundamental Research Funds for the Central Universities and the Ordinary University Graduate Student Scientific Research Innovation Project of Jiangsu province (KYZZ15_0064). We thank all individuals who participated in this study.

\section{Disclosure}

The authors report no conflicts of interest in this work.

\section{References}

1. Schmid AA, Kroenke K, Hendrie HC, Bakas T, Sutherland JM, Williams LS. Poststroke depression and treatment effects on functional outcomes. Neurology. 2011;76(11):1000-1005.

2. Pompili M, Venturini P, Campi S, et al. Do stroke patients have an increased risk of developing suicidal ideation or dying by suicide? An overview of the current literature. CNS Neurosci Ther. 2012;18(9):711-721.

3. Altieri M, Maestrini I, Mercurio A, et al. Depression after minor stroke: prevalence and predictors. Eur J Neurol. 2012;19(3):517-521.

4. Srivastava ATA, Gupta A, Murali T. Post-stroke depression: prevalence and relationship with disability in chronic stroke survivors. Ann Indian Acad Neurol. 2010;13(2):123-127.

5. Zhang T, Wang C, Liu L, et al. A prospective cohort study of the incidence and determinants of post-stroke depression among the mainland Chinese patients. Neurol Res. 2010;32(4):347-352.
6. Zhang N, Wang CX, Wang AX, et al. Time course of depression and one-year prognosis of patients with stroke in mainland China. CNS Neurosci Ther. 2012;18(6):475-481.

7. Narushima K, Kosier JT, Robinson RG. Preventing poststroke depression: a 12-week double-blind randomized treatment trial and 21-month follow-up. J Nerv Ment Dis. 2002;190(5):296-303.

8. Fedoroff JP, Starkstein SE, Parikh RM, Price TR, Robinson RG. Are depressive symptoms nonspecific in patients with acute stroke? Am J Psychiatry. 1991;148(9):1172-1176.

9. Turner A, Hambridge J, White J, et al. Depression screening in stroke: a comparison of alternative measures with the structured diagnostic interview for the diagnostic and statistical manual of mental disorders, fourth edition (major depressive episode) as criterion standard. Stroke. 2012;43(4):1000-1005.

10. Morris PL, Shields RB, Hopwood MJ, Robinson RG, Raphael B. Are there two depressive syndromes after stroke? J Nerv Ment Dis. 1994; 182(4):230-234.

11. Ferro JM, Caeiro L, Santos C. Poststroke emotional and behavior impairment: a narrative review. Cerebrovasc Dis. 2009;27:197-203.

12. Lees R, Stott DJ, Quinn TJ, Broomfield NM. Feasibility and diagnostic accuracy of early mood screening to diagnose persisting clinical depression/anxiety disorder after stroke. Cerebrovasc Dis. 2014;37(5): 323-329.

13. Astrom M, Adolfsson R, Asplund K. Major depression in stroke patients. A 3-year longitudinal study. Stroke. 1993;24(7):976-982.

14. Robinson RG, Jorge RE. Post-stroke depression: a review. $A m J$ Psychiatry. 2016;173(3):221-231.

15. Desmond DW, Remien RH, Moroney JT, Stern Y, Sano M, Williams JB. Ischemic stroke and depression. J Int Neuropsychol Soc. 2003;9(3):429-439.

16. Ayerbe L, Ayis S, Rudd AG, Heuschmann PU, Wolfe CD. Natural history, predictors, and associations of depression 5 years after stroke: the South London Stroke Register. Stroke. 2011;42(7):1907-1911.

17. Rodin G, Voshart K. Depression in the medically ill: an overview. Am J Psychiatry. 1986;143(6):696-705.

18. House A, Dennis M, Mogridge L, Warlow C, Hawton K, Jones L. Mood disorders in the year after first stroke. Br J Psychiatry. 1991;158: 83-92.

19. Cumming TB, Churilov L, Skoog I, Blomstrand C, Linden T. Little evidence for different phenomenology in poststroke depression. Acta Psychiatr Scand. 2010;121(6):424-430.

20. Yue Y, Liu R, Lu J, et al. Reliability and validity of a new post-stroke depression scale in Chinese population. J Affect Disord. 2015;174: 317-323.

21. American Psychiatric Association. Diagnostic and Statistical Manual of Mental Disorders - DSM-5. Washington, DC: American Psychiatric Press, Inc.; 2013.

22. Spalletta G, Robinson RG. How should depression be diagnosed in patients with stroke? Acta Psychiatr Scand. 2010;121(6):401-403.

23. da Rocha e Silva CE, Alves Brasil MA, Matos do Nascimento E, de Braganca Pereira B, Andre C. Is poststroke depression a major depression? Cerebrovasc Dis. 2013;35(4):385-391.

24. Maj M. When does depression become a mental disorder? $\mathrm{Br} J$ Psychiatry. 2011;199(2):85-86.

25. Robinson RG, Bolduc PL, Price TR. Two-year longitudinal study of poststroke mood disorders: diagnosis and outcome at one and two years. Stroke. 1987;18(5):837-843.

26. Carota A, Berney A, Aybek S, et al. A prospective study of predictors of poststroke depression. Neurology. 2005;64(3):428-433.

27. Tateno A, Kimura M, Robinson RG. Phenomenological characteristics of poststroke depression: early- versus late-onset. Am J Geriatr Psychiatry. 2002;10(5):575-582.

28. Paradiso S, Ohkubo T, Robinson RG. Vegetative and psychological symptoms associated with depressed mood over the first two years after stroke. Int J Psychiatry Med. 1997;27(2):137-157.

29. Sibon I, Lassalle-Lagadec S, Renou P, Swendsen J. Evolution of depression symptoms following stroke: a prospective study using computerized ambulatory monitoring. Cerebrovasc Dis. 2012;33(3):280-285. 
30. Robinson RG. Poststroke depression: prevalence, diagnosis, treatment, and disease progression. Biol Psychiatry. 2003;54(3):376-387.

31. Gabaldon L, Fuentes B, Frank-Garcia A, Diez-Tejedor E. Poststroke depression: importance of its detection and treatment. Cerebrovasc Dis. 2007;24(suppl 1):181-188.

32. Chen Y, Patel NC, Guo JJ, Zhan S. Antidepressant prophylaxis for poststroke depression: a meta-analysis. Int Clin Psychopharmacol. 2007; 22(3):159-166.
33. Flaster M, Sharma A, Rao M. Poststroke depression: a review emphasizing the role of prophylactic treatment and synergy with treatment for motor recovery. Top Stroke Rehabil. 2013;20(2):139-150.

34. Van de Meent H, Geurts AC, Van Limbeek J. Pharmacologic treatment of poststroke depression: a systematic review of the literature. Top Stroke Rehabil. 2003;10(1):79-92.

\section{Publish your work in this journal}

Neuropsychiatric Disease and Treatment is an international, peerreviewed journal of clinical therapeutics and pharmacology focusing on concise rapid reporting of clinical or pre-clinical studies on a range of neuropsychiatric and neurological disorders. This journal is indexed on PubMed Central, the 'PsycINFO' database and CAS, and is the official journal of The International Neuropsychiatric Association (INA). The manuscript management system is completely online and includes a very quick and fair peer-review system, which is all easy to use. Visit http://www.dovepress.com/testimonials.php to read real quotes from published authors.

Submit your manuscript here: http://www.dovepress.com/neuropsychiatric-disease-and-treatment-journal 